\title{
SPIN DYNAMICS ON PERCOLATING NETWORKS
}

\author{
G. Aeppli", H. Guggenheim " and Y. J. Uemura" \\ "AT\&T Bell Laboratories, Murray Hill, NJ 07974 \\ \# Brookhaven National Laboratory, Upton, NY 11973
}

\begin{abstract}
.........

We have used inelastic neutron scattering to measure the order parameter relaxation rate $\Gamma$ in the dilute, two-dimensional Ising antiferromagnet $R_{2} C_{0} M_{B_{1-c}} F_{4}$ with $c$ very close to the magnetic percolation threshold. Where $x$ is the inverse magnetic correlation length, $\Gamma \sim \kappa^{2}$ with $z=2.4_{-0.1}^{+0.2}$. Our results are discussed in terms of current ideas about spin relaxation on fractals.
\end{abstract}

The static and dynamic behavior of pure magnets near phase transitions is well established, both experimentally and theoretically.' There also exists a large body of work dealing with the static critical properties of simple disordered magnets. ${ }^{2}$ There are certain random spin systems for which static properties are more difficult to calculate. These systems, ${ }^{3}$ where there are couplings between spins or local fields of random sign, are characterized by "frustration", the inability at low temperatures of certain spin clusters to come into unique alignment with respect to other clusters. Much of the qualitatively new behavior of frustrated systems is dynamical, and certain explanations ${ }^{4}$ of their apparently static properties use dynamical theories as their starting points, thus reversing the pattern followed in the study of critical phenomena in pure systems. In this context, it is natural to ask whether qualitatively new effects arise in simpler, disordered systems, namely systems without frustration. An indication that interesting dynamical behavior might occur in random systems without frustration comes from studies of diffusion in spaces of non-integral (fractal) dimensionality. ${ }^{5}$ For ordinary Euclidean spaces, the mean square distance $\left\langle r^{2}(t)\right.$ خ travelled by a particle in time $t$ obeys the law

$$
\left\langle r^{2}(t)\right\rangle-t \text {. }
$$

In fractal spaces, signals propagate via more circuitous routes than the straight line paths available in normal Euclidean spaces. Thus, particles will require a longer time to diffuse a certain distance in fractal spaces than in the (Euclidean) imbedding spaces.

Correspondingly, the law (1) is generalized to

$$
\left\langle\mathrm{r}^{2}(\mathrm{t})\right\rangle-\mathrm{t}^{2 /(2+\theta)}
$$

with $\theta>0$.

The essential idea of our experiments is to probe dynamical effects on fractals by studying the relaxation of spins located on a fractal. Arguably the simplest fractal is the 
percolatins cluster, realized in magnetic compounds containing sites occupied at random either by non-magnetic ions, such as $\mathrm{Mg}^{2+}$, or magnetic ions, such as $\mathrm{Co}^{2+}$, coupled only to their nearest neighbors. ${ }^{2}$ The exchange couplings in many of these compounds are such that frustration does not arise; while the randomness has profound geometrical effects, it does not lead to a qualitatively new ground state as in the spin glass problem. For our first study ${ }^{6}$ of dynamical effects near percolation, we have chisen $R_{2} C_{0} M_{B_{1-c}} F_{4}$, a system for which the static spin correlations have been studied in du: ail. ${ }^{7}$ Furthermore, there exist experiments ${ }^{8}$ on the dynamical critical behavior of the corresponding pure system, $R b_{2} C_{0} F_{4}$, which is a nearly ideal realization of a two-dimensional Ising antiferromagnet.

We performed our experiments using two triple-axis spectrometers at the Brookhaven high-flux beam reactor. The first of these is located at a thermal beam port and is equipped with a single-pass monochromator. Spectra were collected by varying the final energy $\mathbf{E}_{f}$ with the incident energy $E_{i}$ fixed at $3.5 \mathrm{meV}$; the corresponding energy resolution was $64 \mu \mathrm{eV}$, full width at half maximum (FWHM). The other spectrometer has a double crystal monochromator and is installed at the cold neutron source of the HFBR. Here, we scanned the incident neutron energy $E_{i}$ with the final energy fixed at $2.5 \mathrm{meV}$; the resolution was 37 нeV FWHM.

Our sample was a single crystal of $R b \mathrm{Co}_{c} \mathrm{Mg}_{1-c} \mathrm{~F}_{1}$ with $c=0.58$, which is very close to the nearest neighbor percolation threshold $\left(c-c_{p}-0.593\right)$ for a square lattice. We collected inelastic spectra at a reciprocal lattice point lying on the line (in reciprocal space) where the Bragg condition for two-dimensional antiferromagnetic order is satisfied. Thus, our experiment probes the fluctuations in the staggered magnetization, which has zero mean at all temperatures because $c$ is slightly below $c_{p}$.

Our spectra are well described by Lorentzian profiles which correspond to simple exponential autocorrelation functions for the staggered magnetization $M(t)$,

$$
<M(t) M(t+r)>-\exp -\Gamma|\tau| \text {. }
$$

Because we expect $\Gamma$ for a classical paramagnet - i.e. a collection of decoupled magnetic ions - to approach 0 according to the law $\Gamma-T$ as $T \rightarrow 0$, we show in Fig. 1 the reduced relaxation rate $\mathrm{\Gamma} / \mathrm{kT}$ as a function of the inverse correlation length $\mathrm{ka}$ established by Cowley and coworkers ${ }^{7}$ for the same sample. The data are well-represented by the power law

$$
\Gamma / k T=A(k a)^{2}
$$

where $\mathrm{z}=2.4_{-0.1}^{+0.2}$.

Our value for 2 is very bigh when compared with that $(-1.7)$ found for pure $\mathbf{R b}_{2} \mathrm{CoF}_{4}{ }^{8}$ However, the result that $z$ is high is in accord with the qualitative content of Eq. (2), namely that disturbances on ramified, fractal structures require longer times to travel fixed (Euclidean) distances than they do in normal Euclidean spaces. In our first paper on this subject, ${ }^{6}$ we suggested that the fractal geometry of percolating networks changes Ising spin dynamics in the same manner as it changes diffusion. Furthermore, we posited that the nonlinearities presunt in the spin relaxation, but not the particle diffusion problem, are

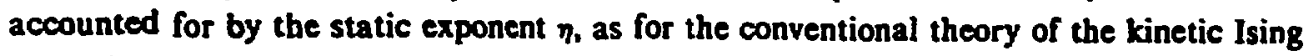
model. 9 We consequently guessed that for fractals,

$$
2=2+\theta-\eta \text {. }
$$

where $\theta$ also occurs in Eq. (2). Using $\theta$ and $\eta$ appropriate to the two-dimensional percolation problem, $z=2.47$, in good agreement with the experimental value. Several theories ${ }^{10,11}$ of spin relaxation near percolation have subsequently appeared. Most of these ${ }^{10}$ use Eq. (4) as a starting point and yield expressions similar to (S) for $z$. Very recently, however, Henley,"I Harris and Stincheombe ${ }^{12}$ have made the important obcervation that Eq. (4) may be incorrect for Glauber models on percolating networks. Instead, they propose a temperature-dependent exponent,

$$
z_{e=1}(T)-2 Z / T \text {. }
$$




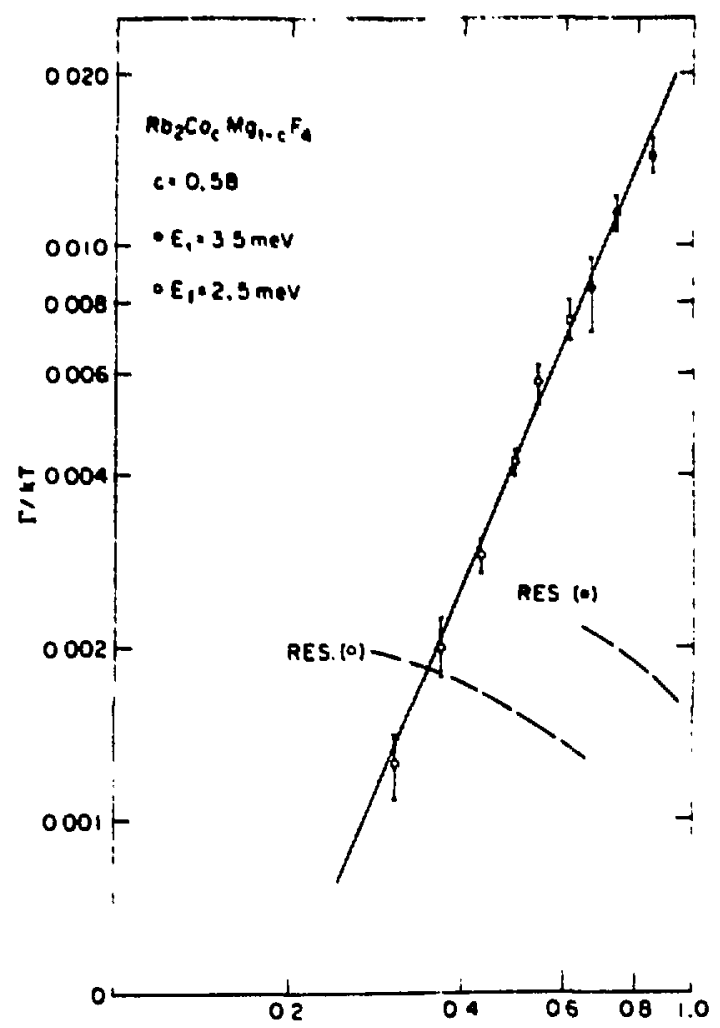

Fig. 1. Normalized relaxation rate $\Gamma / k T$ plotted against reduced inverse correlation $\times a$. At the dashed curves, the FWHM of the resolution function exceeds $\Gamma$ by a factor of 4 . The open and filled circles correspond to the two different spectrometers described in text.

where $Z$ is a geometrical parameter for the fractal and $T$ is expressed in units of the exchange coupling. The derivation of (6) is based on considerations of the energy barriers dominating the low temperature dynamics. These barriers rise logarithmically with the size $L$ of the system, so that

$$
\Gamma \sim \exp \left(-E_{b} / k T\right)-L^{2-(T)} \exp \left(-E_{0} / k T\right)
$$

where $E_{o}$ is an L-independent contribution to $E_{b}$. According to Eq. (7), there should be curvature in a double logarithmic plot of $\Gamma / k T$ against $\alpha \mathrm{a}$, since for Ising systems, $\ln (x a) \sim k T$. Curvature of this nature cannot be discerned in Fig. 1. Unfortunately, due to experimental limitations (see the dashed lines in the figure), we could not measure $\Gamma$ for $\times a<0.3$. However, we anticipate that neutron backscattering experiments ${ }^{13}$ currently in progress at Brookhaven will permit a more stringent test of Eq. (7).

\section{ACKNOWLEDGEMENTS}

We are grateful to $C$. Henley for stimulating conversations and a copy of Ref. 11 to publication, and R. B. Stinchcombe for informing us of his work with C. K. Harris. The work at Brookhaven National Laboratory was supported by the Division of Materials Science, U.S. Department of Energy, under contract No. DE-AC02-76CH00016. 


\title{
REFERENCES
}

I. See, for example, S.-K. Ma, Modern Theory of Critical Phenomena (Benjamin; Reading, MA, 1976).

2. A review of experiments on the percolation and random field problems is given by R. J. Birgeneau, R. A. Cowley, G. Shirane and H. Yoshizawa, J. Stat. Phys. 34, 817 (1984). The theory of percolation is reviewed by D. Stauffer, Phys. Rep. 54, T(1979).

3. An early overview is Ill-condensed Matter, ed. R. Balian, R. Maynard, and G. Toulouse (North Hol and-World Scientific; Amsterdam-Singapore, 1983).

4. H. Sompolinsky and A. Zippelius, Phys. Rev. B25, 6860 (1982).

5. R. Rammal and G. Toulouse, J. Phys. (Paris) Lett. 44, L13 (1983); Y. Geffen, A. Aharony and J. Alexander, Phys, Rev. Lett. 50, 77 (1983).

6. G. Aeppli, H. Guggenheim, and Y. J. Uemura, Phys. Rev. Lett. 52, 942 (1984).

7. R. A. Cowley, R. J. Birgeneau, G. Shirane, H. G. Guggenheim and H. Ikeda, Phys. Rev. B21, 4038 (1980).

8. M. T. Futchings, H. Ikeda, and E. Janke, Phys. Rev. Lett. 49, 386 (1982).

9. P. C. Hohenberg and B. I. Halperin, Rev. Mod. Phys. 49. $4 \overline{35}$ (1977).

10. D. Kumar, Phys. Rev. B30, 2961 (1984); J. M. Luscombe and R. C. Desai, preprint.

11. C. Henley, preprint (1985).

12. C. K. Harris and R. B. Stinchcombe, to be published, and R. B. Stinchcombe, this volume.

13. A. Heidernann, J. D. Ȧxe, L. Passell, D. Moncton, H. Guggenheim and G. Aeppli, private communication.

\section{DISCLAIMER}

\begin{abstract}
This report was prepared as an account of work sponsored by an agency of the United States Government. Neither the United States Government nor any agency thereof, nor any of their employees, makes any warranty, express or implied, or assurnes any legal liability or responsibility for the accuracy, completeness, or usefulness of any information, apparatus, product, or process disclosed, or represents that its use would not infringe privately owned rights. Reference herein to any specific commercial product, process, or service by trade name, trademark, manufacturer, or otherwise does not necessarily constitute or imply its endorsement, recommendation, or favoring by the United States Government or any agency thereof. The views and spinions of authors expressed herein do not necessarily state or renect those of the United States Government or any agency thereof.
\end{abstract}

